Chapter 13

\title{
Optimization of WDM-POF Network for In-Car Entertainment System
}

\author{
Mohammad Syuhaimi Ab-Rahman, Hadi Guna and \\ Norhana Arsad
}

Additional information is available at the end of the chapter

http://dx.doi.org/10.5772/59238

\section{Introduction}

Recent advances in In-Car Entertainment System will play an important role on automotive industry. It was assumed that in the year 2015, every new car, especially built in Europe will be equipped with Internet connection. As cars become connected to the Internet, the demand for Internet-based entertainment and applications and services increases [1].

Polymer optical fibers (POFs) are in a great demand for the data transmission and processing of optical communications compatible with the Internet, which is one of the fastest growing industries in automotive field. POFs become replacement for copper cable technology for future IVI system.

In this chapter reports experimental demonstration of a POFs based solutions in wavelength division multiplexing (WDM) network and some effects due to the placement of color filters as a demultiplexer for the In-Car Entertainment System. A weakly fused (WF) and a highly fused (HF) POF star coupler based on fusing and combining a group of POFs are designed and experimentally investigated. A low-cost demultiplexer is realized by using color filters. The specialized designed plastic-based interference films are used to filter out any other wavelength (color) that is not within the range. Variation of temperature were applied directly to the fused tapered region and an LED fiber source is launched into the fiber input, while the optical power deviation is measured at each output port. The effect of the return optical power and the coupling ratio to the temperature variation of $20^{\circ} \mathrm{C}$ to $125^{\circ} \mathrm{C}$ were investigated. The excess loss, $\mathrm{EL}_{0}$ for the $\mathrm{WF}$ and the $\mathrm{HF}$ coupler at $20^{\circ} \mathrm{C}$ is $\sim 2 \mathrm{~dB}$ and $\sim 8 \mathrm{~dB}$, respectively. 
Media Oriented Systems Transport (MOST) is one of the advance IVI system provider company which also utilized a POF-LED technology to transmit numerous signals represent a different data transmission via time division multiplexing (TDM) network (refer Fig. 1).

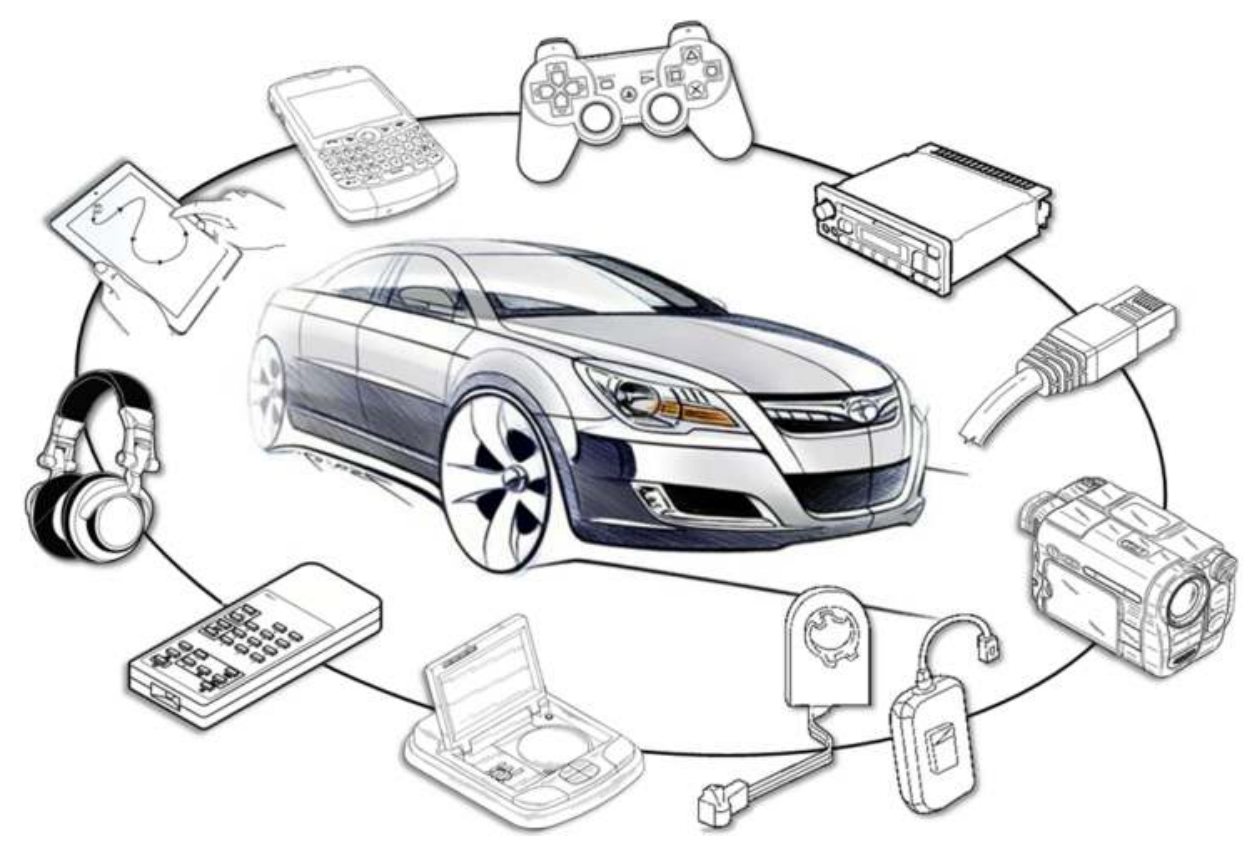

Figure 1. Recent technology on ring topology IVI system with single line of optical fiber cable facing main problem with no backup line when failure occurred.

In our research, we offered a wavelength division multiplexing (WDM) communication network over POF due to the rapid increase of traffic demands [2,3]. WDM is the network that allows the transmission of multimedia data in IVI system over multiple wavelength (color) and thus greatly increases the POF's bandwidth. Beside, this network proposes a backup path in order to mitigate a serious breakdown in TDM-based network in IVI system.

Refer to Fig. 2, the proposed WDM-POF system, three unit of transmitters with different color of LED will carry single information simultaneously. For example in IVI network, red LED with $650 \mathrm{~nm}$ wavelength modulated with video signal while blue $(\lambda 1)$, green $(\lambda 2)$, and yellow ( $\lambda 3$ ) lights carry ethernet, audio and RF signal, respectively. The light has to be combined by the multiplexer (MUX) at the sending side. And to separate the wavelength channels at the receiver side, a wavelength demultiplexer (DEMUX) has to be used.

Demultiplexing, perform the reverse process with the same WDM techniques, in which the data stream with multiple wavelengths decomposed into multiple single wavelength data streams. POF coupler has similar function, operates to combine a number of optical data pulses 


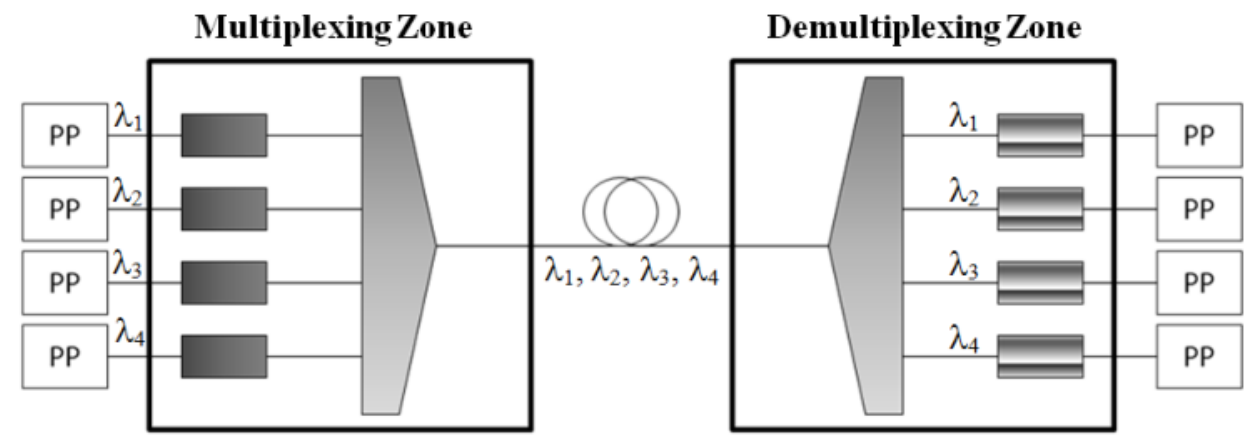

Optical Components:

PP Terminal Instrument

WDM Transmitter

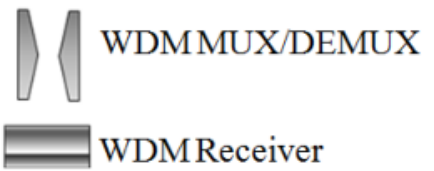

Figure 2. Simple schematic of a 4-channels WDM system.

as a single coupled signal. Hence, the development of MUX based on POF coupler is possible. A low-cost solution for POF-WDM system application will be presented.

A novel fused POF couplers has been fabricated by a fusion technique, as an effective transmission media to split and couple numerous different wavelengths which represents different signals. These novel coupler, however, suffer from several disadvantages. The high cost of the available couplers was raised as a challenge to the development of WDM systems in shorthaul networks [5-8]. In addition, from the stand point of device design, the diameter of the fused tapered region, where stress is concentrated, is too small in conventional couplers. The structure causes a high incidence of fiber fracture, which results in poor reliability [9-12].

Thus a cost-effective $3 \times 3$ POF couplers based on a fused tapered structure to address these drawbacks of conventional couplers is demonstrated. The coupler is fabricated by a new and simple fabrication method, using a Bunsen burner and a metal tube. In this study, two types of fused couplers method focused of weakly fused (WF) and highly fused (HF). The WF coupler is, however, not considered to be a low-loss device, as the excess loss of the coupler was high, 12 to $22 \mathrm{~dB}$.

The HF coupler is then developed to be the successor of the WF coupler. The excess loss of the HF coupler is very low, 0.3 to $5 \mathrm{~dB}$. The device is developed as an optical switch which optical power can be switched completely from one fiber to another fiber at a temperature increase of $\mathrm{T}=55^{\circ} \mathrm{C}[13]$. The switching characteristic can be achieved by varying the refractive index of the cladding at the coupling region of the coupler by temperature.

One of the aim of this chapter is to examine and optimize the feasibility of both methods of $3 \times 3$ POF couplers as thermal optical switches to be integrate in WDM-POF-based network for IVI system. The investigation is also to determine whether the thermal treatment is required 
to improve the quality and shift the device specification. Hence, a study of thermal effect on both polymer-based WF and HF couplers by varying the temperature of a hot plate from 20 ${ }^{\circ} \mathrm{C}$ to $125^{\circ} \mathrm{C}$ is studied. The fused tapered fiber in the coupling region is exposed to the hot plate surface and optical power is launched into the input fiber of the coupler. In this temperature-dependence experiment, we investigate a relationship between temperature and several parameters such as coupling ratio, insertion loss and excess loss of the couplers.

In this chapter, red LED $(650 \mathrm{~nm})$ has been utilized to transmit Ethernet data while green LED (520nm) can transmit a video image generated from CCTV network or DVD player, and blue LED with 470nm wavelength represents an audio transmission system for home networking. Refer to Fig. 3, special polymer color filters has been located between the coupler and receiverend to ensure the entire WDM system can select a single signal as desired [4].
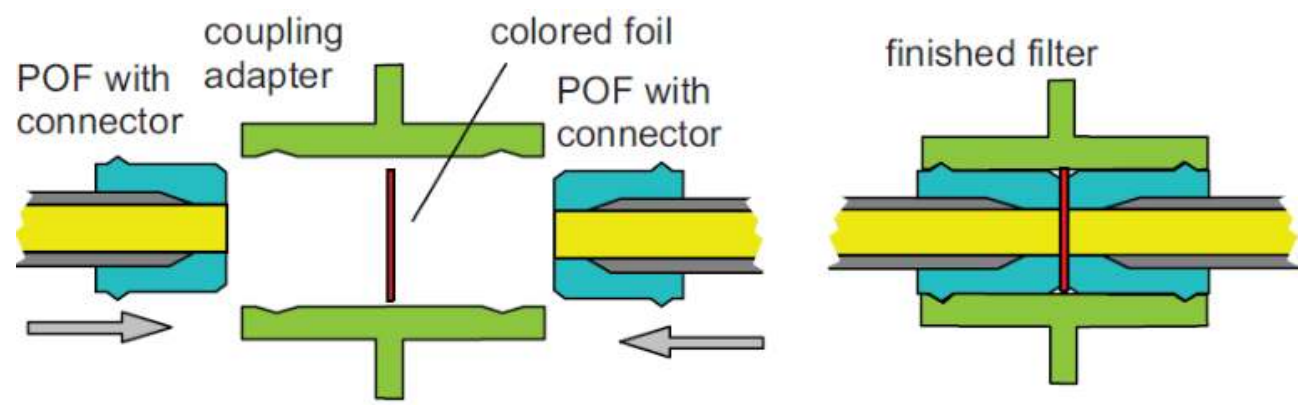

Figure 3. Each of those color filter lets pass exactly one wavelength and reflects all the others. By using several of those filters, the different wavelengths can be sorted out in a very low-cost way.

The performance of the novel coupler either with or without attachment of color filter can be evaluated in terms of insertion loss (IL). Some experiments in order the optimized the performance of WDM-POF based system for IVI system need to be conduct to minimized the value of the insertion loss (IL) in the network. The insertion loss (IL) is the amount of power loss that arises in the fiber optic line from input to the output of the fabricated coupler, expressed below,

$$
I L_{\text {port }}(d B)=10 \log \frac{P_{\text {oport }}}{P_{i}}
$$

Some wavelengths interfere with their reflected parts constructively, whereas others interfere destructively. Those wavelengths that interfere constructively can pass the filter, whereas the others get reflected. Besides the material parameters, the incident angle plays a major role, as each layer gets relatively thicker when tilting the filter. Color filters are manufactured for a long time and therefore high quality filters are readily available. They usually have sizes of several square millimeters. Color filters therefore are a valid choice to build DEMUX. 


\section{WDM-POF integrated network for in-car entertainment system}

Adapting the fused tapering technique for conventional multimode fiber, we successfully established fabrication process for $1 \times 3$ POF twisted and fused couplers to be used as a MUX and DEMUX in IVI system. The $1 \times 3$ low cost coupler is an optical device, which ended by 3 number of POF output ports, while the other side ended by one POF port.

Similar to common coupler, it is also possible to work bidirectional, whereby it works from the 3 ports into 1 port (for coupling signal purpose), or vice versa (for splitting signals purpose). Optical $3 \times 3$ coupler has been symmetrically cut into two part to generate a pair of $1 \times 3$ couplers by the jointing of three polymethylmethacrylate (PMMA) POF [14]. Other specification for the design, the input POF is designed and fabricated to be twisted and fused shape as the fabrication process and $1 \times 3$ POF coupler is illustrated in Fig. 4 .

\section{Induced thermal resistance} (heat capacity)

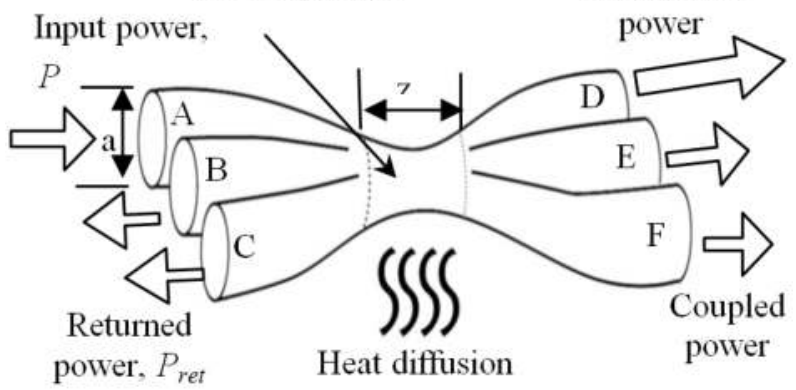

Figure 4. Thermal resistance at the coupling region of POF coupler in which all fibers are fused together.

Standard multimode SI-POF is used with its core diameter of $980 \mu \mathrm{m}$ and cladding thickness of $10 \mu \mathrm{m}$. To obtain the results, DEMUX is realized using a special color filter attached using epoxy resin to the end of the connectors. The components are chosen because they are low cost and are easily found in the market.

Almost similar to POF material itself, the color filters are comprised of two types of plastic. More than $65 \%$ of the line is made from co-extruded polycarbonate plastic. The remainder of the line is deep dyed polyester $[15,16]$. Filters create color by subtracting certain wavelengths of color. Thus, a red filter absorbs blue and green, allowing only the red wavelengths to pass. The process is subtractive not additive, so the light source must emit a full spectrum.

The swatch book provides detailed information on the spectral energy curve of each filter. The curve describes the wavelengths of color transmitted through each filter. For example, Supergel 342 transmits approximately $40 \%$ of the violet and blue energy of the spectrum and $75 \%$ of the orange and red energy. It absorbs all energy in the yellow and green range $[15,16]$. 
After putting the resin onto the filter to be attached to the socket, the component then is hold together tightly for about two minutes to assure that no gap or air bubbles all over and also to assure the strong bond. This part has to be done gently since the epoxy resin has to be avoided covering the fiber's surface as much as possible so that any power losses can be minimized when the measurement is taken. However, since the edge of the socket is quite thin and sharp, the spread of the epoxy resin to the fiber surface cannot be $100 \%$ avoided.

After the fabrication is done, readings and measurements are taken for insertion loss for each of the fiber using a power meter. In this experiment, a lot of samples were fabricated to get the optimal results and to see which of the color filters that shows the most transmission and gives least losses. The length of the POFs is fixed at 3 meters long.

In this study, for fused plastic optical fiber, the optical loss is categorized as extrinsic loss due to the physical change of POF, LED projection to POF and the core-to-core connection and [17, 18]. It is obtained that the physical change of POF caused by fabrication process, where by diameter of POFs increasingly decrease to approach $1 \mathrm{~mm}$ and the POFs finally has twisted and fused shape. In characterization process, optical loss may present through the direct LED projection to POF surface. Besides, optical loss may also present through the connection between the fused tapered POF and POF cable [17].

\section{Results and discussion}

Comparison for the optical line either using the color filter or not, has been analyzed. The insertion loss of as much as 21 samples for the output terminal over POF has been utilized in 3-channels IVI system through red filter (internet data) have been visualized in Fig. 5. From the results gathered, it is seen that when all the components are set up and red LED (650nm) was injected, the insertion loss measured by the power meter shows small increase of losses when the film is attached to the socket.

This is also true when blue and green LEDs are injected. For the characterization of same film using different sources, we take a red film A (filter labeled \#4690) as the primary filter and it is injected with all three LEDs, red, green and blue transmitters. As the results depict, the red filter injected with red transmitter shows a small increase of losses which averagely $I L<\sim 3 \mathrm{~dB}$ compared to the initial loss before the film is attached to the fiber. Same goes to the power output when the film is attached to the fiber.

The effect of plastic-based filter attachment at the receiver-end did not indicate a significant deviation in power efficiency since both PMMA and Co-extruded Polycarbonate for fiber core and plastic filter's material have the same refractive index which approaching 1.59 [16].

Study on the saturation level of each color filters - red, green and blue - has been carried out. As much as eleven best samples chosen with different level of color saturation labeled from 1 to 11 which sample number 1 indicate the darkest (more saturated) color filter while number 11 with the lightest color (less saturated). Each of different color filter has been injected by LED in a range of blue to red light $(470 \sim 650 \mathrm{~nm})$ and the result can be obtained in Fig. 6 . 


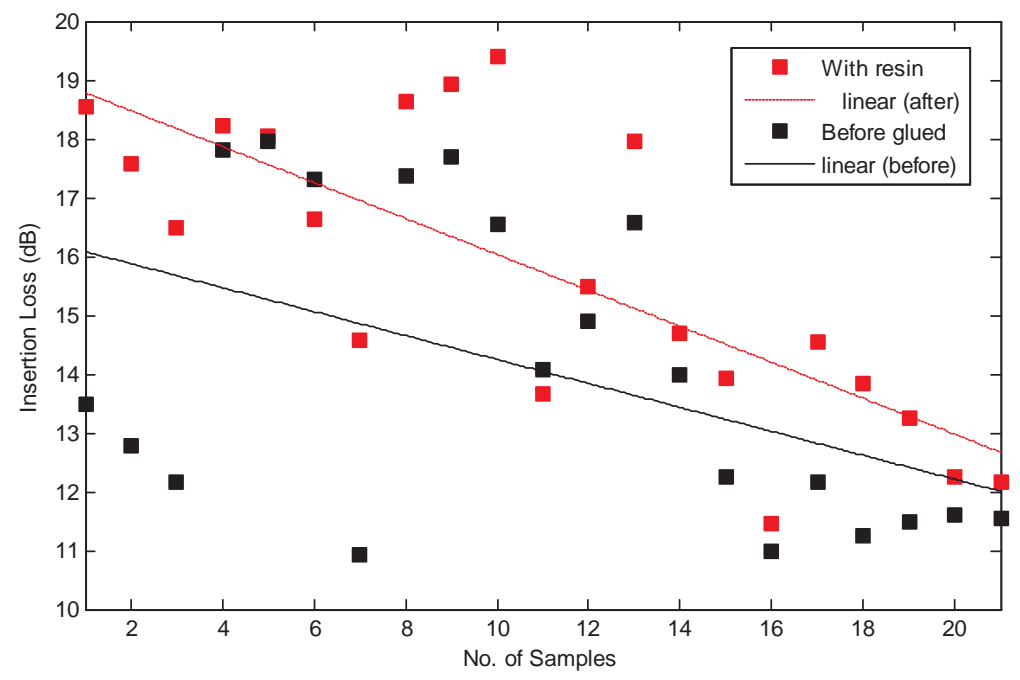

Figure 5. Effect on resin for demultiplexer filters approximately $1 \mathrm{~dB}$ insertion loss occurred on the measurement between before and after connector glued by resin.

From the result in Fig. 6(a), it is observed that sample 1 to 7 shows highest losses and decrease of efficiency. Since sample 1 to 7 being among the darkest film color meaning that only small or narrow transmission percentage of red LED or transmitter is allowed to get through. This phenomena also found in Fig 6(b), for the first six sample has quite high losses especially when it was injected by LED in blue to green range (470-580nm). However, red filter in Fig 6(c) did not indicate a significant deviation of loss by variying the saturation of the filter.

Compare with others, red filters block most of the green and blue LED transmission since it is clear that only red wavelength $(\lambda=600 \sim 650 \mathrm{~nm})$ will be allowed to get through the film. The film filters out any other wavelength transmission that is not within the range. This concept is used as the primary idea for designing the demultiplexer.

It has been proven that, when blue filter was injected by all three optical source (blue, green and red color) all the data were more fluctuated right after the light hit the filter through the fiber. Same goes to green filter, all light sources will fluctuated the efficiency of the data transmission. This fluctuation generated as an effect from the SED percentage of each filters and also came from the intensity of each light source. The higher intensity of the light source transmitted the more oscillated graph plotted and the less significant of SED percentage deviation the less effect on fluctuation.

For the temperature-dependence experiment, two samples of $3 \times 3$ fused couplers with multimode SI PMMA POF are tested: WF and HF couplers. For the WF coupler, the fabrication method includes three processes: fiber bundle configuration, fabrication of spiral fiber and fiber tapering. Firstly, a fiber bundle consists of $N$ core unjacketed polymer fibers (where $N=3$ ) 
is inserted into the metal tube and placed in symmetrical coordination. The fibers bundle is exposed to the heat of the flame repeatedly twisted while pulled continuously to produce a spiral fiber with a particular number of twists in the centre of the fiber bundle. The length of the fused region is limited to $8 \mathrm{~cm}$. While the indirect heating process continues, fiber bundle is pulled and twisted. When the POFs reach the melting point, the fiber bundle is pulled to fuse the fibers, and the fused tapered fiber is formed. Each fiber ports (output and input) are insulated with PVC sleeve.

The HF coupler fabrication method includes four processes, as a new process was introduced to enhance the coupling characteristic of the fused tapered fiber by removing twisting effects on the fused fiber. A twisting effect implies that each fiber is not melted sufficiently to combine with each other. Firstly, a fiber bundle consisting of $N$ core unjacketed polymer fibers (where $N=3$ ) are inserted into the metal tube and placed in symmetrical coordination. The fibers bundle are pulled and exposed to the heat of the flame repeatedly and continuously to produce a spiral fiber with a particular number of twists in the centre of fiber bundle. The length of the fused region is limited to $5 \mathrm{~cm}$.

The fiber bundle is pulled and twisted from both sides repeatedly and continuously over a long fusion time, $t_{f}<65 \mathrm{~s}$, to reach a sufficiently high melting temperature to remove twisting effects. While indirect heating process continued, fibers bundle is pulled and twisted. When the POFs reach the sufficient melting point, the fiber bundle is pulled to fuse the fiber, and the fused tapered fiber is formed. Each fiber ports (output and input) are insulated with a PVC sleeve.

The experimental setup has been setup, which consisted of a digital hot plate, an AF-OM110A power meter and an LED fiber source with a $650 \mathrm{~nm}$ wavelength. Refer to Fig. 4, each fibers on the left side of the coupler are defined as Port A, B, or C, whereas on the other side, each fiber ports are termed as Port D, E, or F. In the experimental setup, for each coupler, the hot plate is exposed directly to the centre region (the fused and tapered fiber) and the temperature of the hot plate is varied from $30^{\circ} \mathrm{C}$ to $125^{\circ} \mathrm{C}$ to investigate the light propagation behavior and power loss for each fiber port. The LED source is launched into a single input fiber, while each output fibers was connected into an optical power meter using a suitable adapter to measure the output power. In the meantime, another optical power meter is also placed at the end of each input fibers to measure the returned power for both fiber ports. By using the optical power acquired from the measurement, several parameters were calculated for both couplers, such as the excess loss and the coupling ratio.

When the heating temperature is increased to $T>85^{\circ} \mathrm{C}$, the behavior of power dissipation at the other input port differs from that in the first case described above. This phenomenon reveals that the induced thermal resistance in the centre of the coupler reaches a very high resistivity, where large heat capacity stored in the coupling region is sufficient to oppose optical power transferred between input and output fibers. In addition, there is a possibility that the optical power is switched incompletely from output to input fibers. While output power $P_{o}$ in through-put and cross-coupled fibers remains fall to zero, the returned optical power $P_{\text {ret }}$ in other input fiber increase drastically. In this case, the optical power tends to be reflected and coupled to other input fibers, instead of output ports. As the fused tapered fiber 


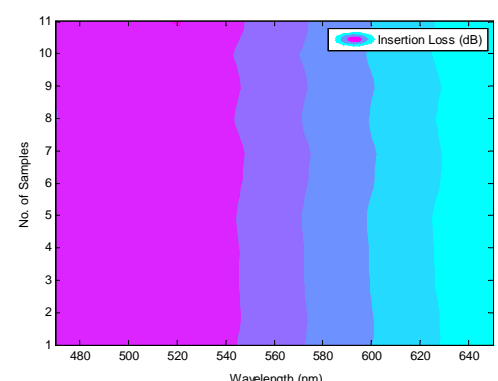

(a)

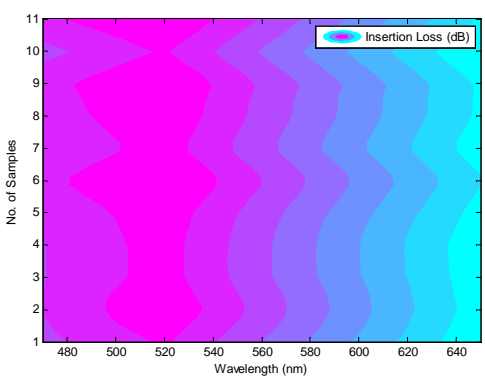

(c)

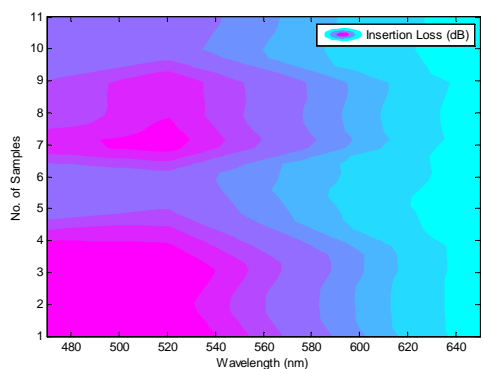

(e)
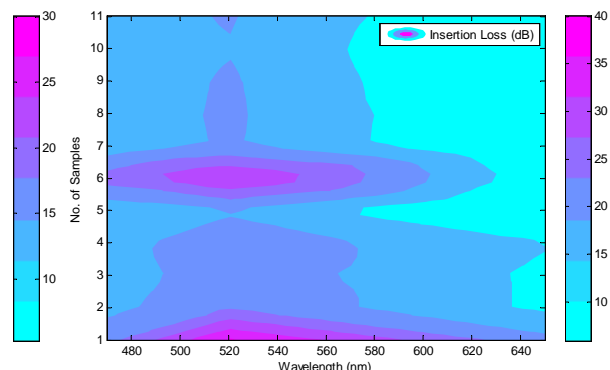

(b)
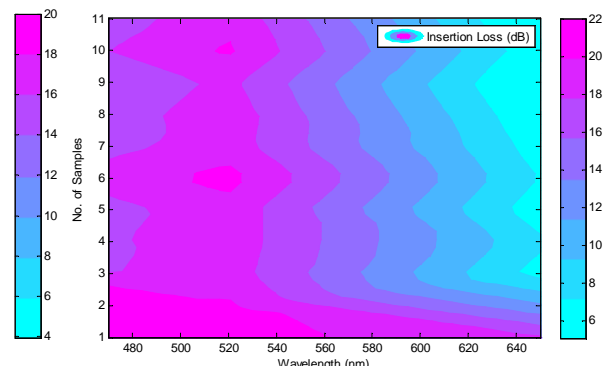

(d)
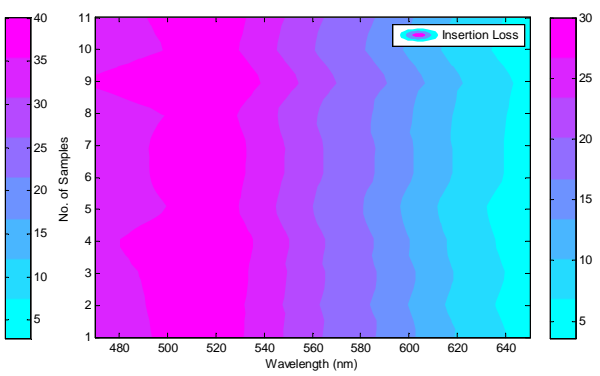

(f)

Figure 6. Comparison on before (left side) and after (right side) effect on injection of different saturation of color filter. Three basic colors have been investigated for (a) blue, (b) green and (c) red filter, before (left side) and after (right side) injected with LED in visible light range $(450 \sim 650 \mathrm{~nm})$.

is overheated $\left(T \gg 85^{\circ} \mathrm{C}\right)$, however, the returned optical power begins to break down as the fused fiber in the coupling region is approaching damage threshold at which the coupler lifetime begins to decrease. The same behavior is also exhibited by using the optical power at the output fibers.

The heating temperature of the hot plate $T$, is increased gradually from $30^{\circ} \mathrm{C}$ to $125^{\circ} \mathrm{C}$ to examine the thermal effect each port of the fibers. Previous experimental results show that the refractive index variation with temperature is analyzed for large-core PMMA POF [13]. The 
following function is used to describe the variation of the refractive index by performing a nonlinear least-square fit: $n(T)=n_{0}+a T+b T^{2}$. The symbol- $\alpha=d n / d T\left({ }^{\circ} \mathrm{C}^{-1}\right)$ denotes the thermal dispersion of the refractive index, where $\alpha=-5 \times 10^{-4}$ for PMMA and $\alpha=-4 \times 10^{-4}$ for fluorinated polymer $[19,20]$, which are the materials of the core and the cladding, respectively. At room temperature $\left(T=20^{\circ} \mathrm{C}\right)$, the refractive index of the core is higher than that the cladding. For typical POF, it has been found that the index for the PMMA core and the fluorinated polymer cladding at $20^{\circ} \mathrm{C}$ are $n_{c o}=1.490$ and $n_{c l}=1.402$, respectively [21]. The refractive indices of the cladding and the core at the fused region exhibit different temperature-dependence behavior. As the heating temperature is increased $\left(T>20^{\circ} \mathrm{C}\right)$, the difference in refractive index between the core and the cladding, $\Delta n=n_{c o}-n_{c l}$ becomes smaller. In comparison, the percentage of the core index reduction is higher than in the cladding material, as the thermal dispersion $d n / d T$ for the PMMA core is greater. As calculated, at temperature $T=100^{\circ} \mathrm{C}$, the refractive indices for both material would be equal $\left(n_{c o}=n_{c l}\right)$ and the difference of the two indices would be zero $(\Delta n=0)$. At this point, the fiber stops confining light to the core, and the output light intensity drops to near zero. In practice, optical loss and/or power dissipation increase gradually; they do not cut off abruptly.

While heat energy is supplied from a hot plate to the coupling region at the fused coupler and an LED fiber source with wavelength of $650 \mathrm{~nm}$ is injected into an input fiber, thermal resistance would be induced at the centre of the fused coupler to oppose light propagation from a single input fiber into multiple output fibers. The symbols $T_{R}$ and $T_{m}$ denote room temperature and melting point temperature, respectively. It is known that $T_{R}=20^{\circ} \mathrm{C}$ and $T_{m}=85^{\circ} \mathrm{C}$. In the experiment, there are two cases of power dissipation occurring within different behavior.

Firstly, when the temperature is increased to $T>20^{\circ} \mathrm{C}$, a small heat capacity is stored in the fused and tapered fiber, while heat energy is distributed along its taper length $z$. In this case, the heat capacity accumulated in the coupling region represents thermal resistance. As the induced thermal resistance is not sufficient to block optical power transmission completely, optical power can pass through coupling region to output fibers with low power intensity. As the coupling region is heated directly, the change in the refractive indices of the core and the cladding result an optical power loss in each of the fiber output port.

The optical power was measured for one directions, the LED fiber source was injected into Port A, while the optical power meter was positioned at the ends of the output fibers (Ports $\mathrm{D}, \mathrm{E}$, and F). The room temperature $T=20$ was taken as a reference value before the experiment was started. In the experiment, the temperature of the hot plate was increased in steps of $5^{\circ} \mathrm{C}$, with a time delay of $60-70 \mathrm{~s}$ to reach a stable condition. Fig 7 shows the influence of varying temperature $T$ from $30{ }^{\circ} \mathrm{C}$ to $125^{\circ} \mathrm{C}$ on output power $P$ o for both fused couplers (HF and WF) wherein the LED fiber was injected into the input fiber from the left side to the right side of the coupler.

As shown in Fig 7(a) and 7(b), in each fiber port, output power decreases as temperature rises. Both types of fused polymer couplers will be damaged when the heating temperature increased to $T=125^{\circ} \mathrm{C}$. In the case of the HF coupler, as shown in Fig 7(a), optical power 
decreased gradually when temperature varied from $30^{\circ} \mathrm{C}$ to $95^{\circ} \mathrm{C}$. At $30^{\circ} \mathrm{C}$, the differences between the transmitted power at Port $\mathrm{D}$ and the coupled power at other output fibers were significantly large. However when the temperature of the hot plate increased to $\mathrm{T}>95^{\circ} \mathrm{C}$, however, the difference became relatively small $(\Delta \approx 0)$ such that the optical power in each output fiber fell down drastically to zero. The temperature point at $95^{\circ} \mathrm{C}$ thus defined as the damage threshold, because the coupler lost temperature stability at this point. As the melting point of the polymer material at $85^{\circ} \mathrm{C}$, it is believed that the polymer fiber suffered from excessive bond rupture at $95^{\circ} \mathrm{C}$.

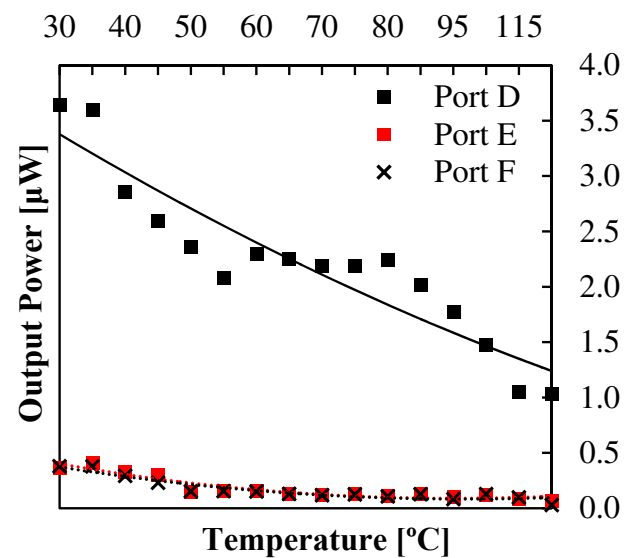

(a)

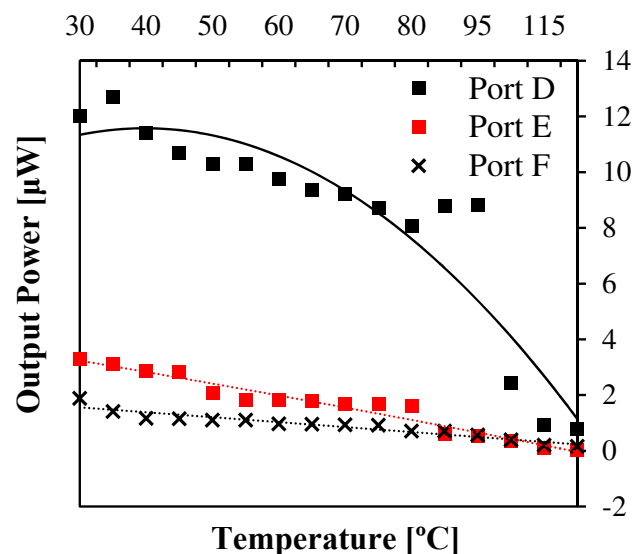

(b)

Figure 7. The relationship between temperature variation $\left(30^{\circ} \mathrm{C}\right.$ to $\left.125^{\circ} \mathrm{C}\right)$ and optical power for (a) the HF coupler and (b) the WF coupler. 
In the case of the WF coupler, as shown in Fig 7(b), the optical power reduction in the crosscoupled fibers (Ports E and F) was not significant, as the power intensities for both cross coupled ports were too small, less than $0.5 \mu \mathrm{W}$ at $30^{\circ} \mathrm{C}$. It was found that the throughput port $\mathrm{D}$ decreased with similar behavior as that seen in the WF coupler, with the power falling to zero suddenly when the temperature of the hot plate increased to $T>95^{\circ} \mathrm{C}$.

However, for temperature variations from $30^{\circ} \mathrm{C}$ to $85^{\circ} \mathrm{C}$, the downward slope for the WF coupler $(-\mathrm{dP} / \mathrm{dT})$ was greater than for the HF coupler. It is believed that the geometrical taper design (in the coupling region) influenced the $-\mathrm{dP} / \mathrm{dT}$ slope. As the twisting effect was featured in the fused tapered fiber in the centre of the WF coupler, it is believed that the large fiber imperfection in the fused fiber region changes the total optical transmission characteristic of the polymer fiber. Therefore, the twisting effect is considered to be a minor factor in determining the power loss in the WF coupler.

Another effect to take into account is the heating time delay that occurred during power measurement for each fiber port. The delay caused the temperature of the polymer material to increase and thus resulted in optical loss. At $T \gg 95^{\circ} \mathrm{C}$, the results show that the optical power $P_{0}$ in each output fiber (Ports D, E, and F) decreased drastically to zero with high $-\mathrm{dP} / \mathrm{dT}$ slope. In this case, it was believed that the refractive indices of the core and the cladding were equal, $\left(n_{c o}=n_{c l}\right)$. As the PMMA core has a large dn/dT coefficient, the core index reduced faster than the cladding index, and as a result, the indices for the core and the cladding became equal at a certain point before the coupler damage occurred.

In addition to the measurement presented above, the returned power $P_{\text {ret }}$ was measured at the other input fibers to investigate the thermal switching behavior the couplers. During the measurement, the optical power meter was placed at the ends of the two other input fibers.

Fig 8 shows the temperature dependence of the coupling ratio for both couplers in their throughput and cross-coupled fiber ports for both directions of lightguide propagation. The coupling ratio in the throughput port is defined as $C R_{T}(\%)=P_{t} / P_{s^{\prime}}$, whereas the cross-coupled port is $C R_{c}(\%)=P_{c} / P_{s}$. The symbols $P_{s}$ and $P_{t}$ denote the transmitted power at the throughput port and the coupled power at the throughput port, respectively.

As both the WF and the HF multimode PPMA POF fiber couplers are wavelength independent, their coupling ratios are not periodic functions. For an ideal wavelength-independent $3 \times 3$ coupler, it is assumed that the fused fiber in the coupling region has a strong coupling; the output power ratio in the throughput fiber and the cross-coupled fibers at room temperature, $T=20{ }^{\circ} \mathrm{C}$, is thus equal to $33 \%$ and $66 \%$, respectively. For practical couplers, however, the couplers are afflicted with large error in the power ratio, as the coupling ratio at the throughput outlet is significantly higher than the power ratio at the cross-coupled port.

For the WF coupler, as shown in Fig 8, the ratio error $\varepsilon$ at $20^{\circ} \mathrm{C}$ is less than $\pm 50 \%$, whereas for the HF coupler, $\varepsilon= \pm 36 \%$. It is believed that, for both couplers, the fused fibers in the coupling zone were not completely fused. In the other words, the fibers were not coupled to each other with a $100 \%$ coupling ratio. The WF coupler has a lower coupling efficiency than the HF coupler, as the HF coupler's coupling ratio error is smaller. It is known that the HF coupler 


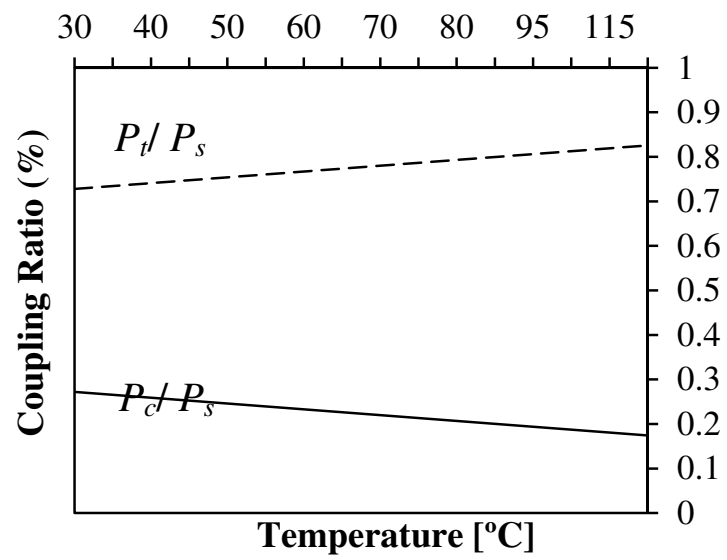

(a)

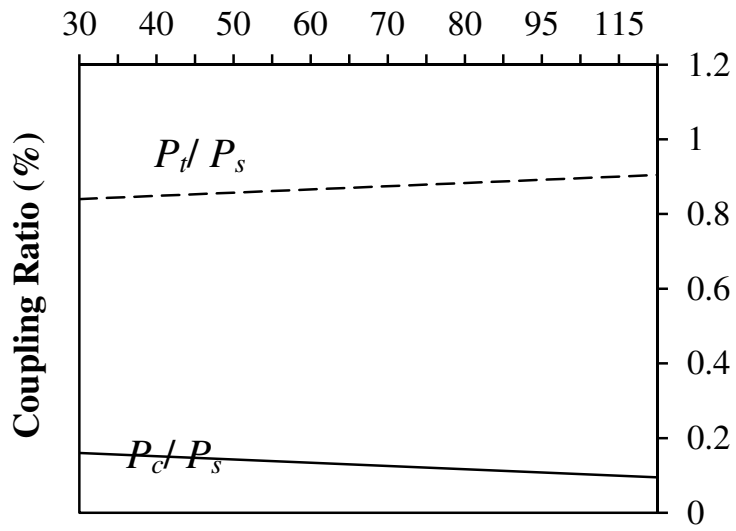

Temperature $\left[{ }^{\circ} \mathrm{C}\right]$

(b)

Figure 8. Coupling ratio variations with temperature increase for (a) HF and (b) WF coupler.

was fabricated to have a plane surface on its fused tapered fiber, with no twisting effect featuring in the structure. The twisting effect is an undesired design characteristic; as the fibers in the coupling region may not be highly fused and thus have low optical power coupled.

As mentioned before, the refractive index of the fused tapered fiber decreases with temperature. The thermal change of the refractive index in the core and the cladding in the coupling region will result in the variation of the relative phase velocity of the interaction light modes, and the coupling ratio of the fused coupler will be thus influenced by rising temperature [22].

As shown in Fig 8, the variation of the coupling ratio for both couplers is a linear function. The experimental result shows that, for both couplers, the ratio of the transmitted power increases 
with rising temperature from $30^{\circ} \mathrm{C}$ to $125^{\circ} \mathrm{C}$, while the ratio of the coupled power decreases in the meantime.

Fig 8(a) indicates that, for the HF coupler, the average ratio of the coupled power reduces from $35 \%$ to $23 \%$, whereas the ratio of the output power in the throughput fiber increases linearly from $68 \%$ to $74 \%$. Fig 8 (b) shows that, in contrast to the HF coupler, the WF coupler's power ratio in the cross-coupled fiber decreases linearly from $18 \%$ to $16 \%$. Moreover, the ratio of the transmitted power increases from $82 \%$ to $86 \%$. As the $\Delta C R / \Delta T$ slope is small, it can be seen that the influence of the temperature effect on the coupling ratio is minor, and the couplers are thermally stable with respect to their coupling ratios.

In this study, at room temperature $\left(T=20^{\circ} \mathrm{C}\right)$, extrinsic loss is considered as major contributing factor to optical loss for both the HF and WF couplers, as the fused fiber in the coupling region was degraded by structural fiber imperfections. These fiber imperfections include the change in fiber diameter from $2.8 \mathrm{~mm}$ to $\sim 1 \mathrm{~mm}$, twisting effects and polymer degradation via liquefaction.

In normal conditions without temperature influence, the power splitting performance of the HF coupler is more significant than that of the WF coupler, as the fused fibers in HF coupler suffer from relatively few imperfections. In spite of the different levels of power loss for the two couplers, the fiber imperfections that are characteristic of the fused fiber regions can be considered a design constant, as the excess losses of both couplers decrease with similar $\triangle E L /$ $\Delta T$ slopes.

On the other hand, the efficiencies of both couplers lie at the same point when the temperature has been increased to $T>95^{\circ} \mathrm{C}$, as both couplers are close to the ends of their lifetimes. Both couplers are destroyed when the temperature is increased to $125{ }^{\circ} \mathrm{C}$. It is realised that the highest temperature to which polymer material may be exposed to the heat while still retaining its structural integrity is $\sim 85{ }^{\circ} \mathrm{C}$ and that the glass transition temperature for $\mathrm{POF}$ is $90{ }^{\circ} \mathrm{C}$. The experimental results show that each coupler lost its temperature stability when its fused tapered fiber is overheated to $T>95{ }^{\circ} \mathrm{C}$, bringing the device close to the end of its lifetime. It is known that supplying thermal energy of $290 \mathrm{~kJ} /$ $\mathrm{mol}-375 \mathrm{~kJ} / \mathrm{mol}$ causes the bond rupture of the polymer chains and thus changes the total performance characteristics of the fused coupler [23, 24].

In the experiment, $95^{\circ} \mathrm{C}$ is thus defined as the PMMA POF damage threshold. In the case of polymer material, the $d n / d T$ coefficient is negative because the material's thermal expansion is higher than the temperature coefficient of the electronic polarisability [25]. Hence, the thermal expansion coefficient is dominant. As the $d n / d T$ coefficients for the PMMA core and the cladding are negative, the refractive indices of both materials decrease with increasing temperature. It is realised that the $d n / d T$ coefficient for the PMMA core is higher than that of the cladding. As a result, the refractive index of the core decreases more rapidly, and the indices of both materials become nearly equal when the temperature increases to $95^{\circ} \mathrm{C}$. At $T \gg 100^{\circ} \mathrm{C}$, the output power in each fiber port suddenly breaks down to zero, as no power reflection and/ or transmission occurs through the medium of the fused fibers. 


\section{Conclusion}

The combination of WDM with POF will broaden the horizon of low cost optical customer premises networks [26]. A technique has been used for fabricating the optical coupler based on POFs technology using multimode SI-POF type with $1 \mathrm{~mm}$ core size. Fabrication and characterization stages have been carried out to develop the coupler [14]. A technique also has been used to develop a demultiplexer for short-haul communication based on plastic optical fibers. This experiment shows the transmission of multiple signals with different wavelengths carried through one fiber. The concept of multiplexer and demultiplexer are the basic of this system. The system only utilizes three colors for the transmitters and also the filters for the demultiplexer which are blue, green and red $(\lambda=430,570$ and $650 \mathrm{~nm})$. Light source from the red, green and blue transmitters are combined by using multiplexer. In order to separate the combined signals, special separators - called demultiplexers (DEMUX) - are utilized. These DEMUX are realized by employing the principle of the Color filters.

Filters play an important role in giving a higher insertion loss from the WDM-POF system, but the quality of a number of output port is not badly destructed due to the color band gap from the filter itself, speed rate of the Internet still stable and the resolution of the video image is quite good. Some parameters, such as optical output power and power losses on the devices were observed, and not to mention about the effect of filter placement and the efficiency of the handmade $1 \times N$ coupler itself.

Red LED with a $650 \mathrm{~nm}$ wavelength has been injected to different Color filters for the purpose of characterization test in order to analyze the level of power efficiency of the demultiplexer. Analysis shows that efficiency maintains for filter of the same wavelength as the transmitter while other range of wavelengths will mostly be filtered out or blocked. This main idea is fully utilized for the designing of demultiplexer for WDM-POF-based IVI-Systems applications. Final analysis shows that efficiency of the filter can reach up to $70 \%$. Improvement of performance can be made through practice. Although the setup IVI system exhibits very high attenuation of the transmission, this concept of handmade optical coupler and demultiplexer has been tested for sending data for video, audio and Ethernet and the output shows successful performance.

In the temperature-dependence experiment, it was proven that thermal resistance exists in the fused tapered fiber at the centre of the coupler. The thermal resistance of the fused fiber is dependent on the heat capacity stored in the coupling region. As the heat capacity of the fused tapered fiber reaches its level of saturation, the internally induced thermal resistance is sufficient to block light propagation from the input fibers. Thus, some portion of the total input power is reflected along the opposite path to the two other input fibers. The resultant light guide propagation is called thermal switching.

Hence, the obtained result reveals that WDM-POF has great potential to be employed as economical wavelength divisions multiplexer because it is able to couple different wavelengths with main advantages that are low optical loss and low cost. An intensive study suggested in order improving the homogeneity of this prototype. In fact, fusion technique 
afflicted with some disadvantages has no consistency of producing coupler as it was almost not possible to fabricate POF coupler with good performance consistently. This WDM-POF technology can be improved gradually through experience and practice. This device is highly recommended for WDM-POF system as it is not as costly as other commercial POF coupler. Furthermore, the fabrication and installation process is simple, easy and suitable to be used for WDM-POF based IVI-system application.

\section{Acknowledgements}

This research has been conducted in Computer \& Network Security Laboratory, Universiti Kebangsaan Malaysia (UKM). This project is supported by Ministry of Science, technology and Environment, Government of Malaysia, 01-01-02-SF0493 and Prototype Research Grant Scheme PRGS/1/11/TK/UKM/03/1. All of the handmade fabrication method of POF coupler, $1 \times \mathrm{N}$ handmade ${ }^{\mathrm{TM}}-\mathrm{POF}$ coupler and also the low cost WDM-POF network solution were protected by patent numbered PI2010700001.

\section{Author details}

Mohammad Syuhaimi Ab-Rahman , Hadi Guna* and Norhana Arsad

*Address all correspondence to: hadi_guna87@yahoo.com

Universiti Kebangsaan Malaysia, Selangor Darul Ehsan, Malaysia

\section{References}

[1] Europe, E. Cars get internet connection, study says, 2008.

[2] Hashimoto, M., et al. Design and Control System over WWW for Regional CWDM Optical IP Networks with Reconfigurable Optical Add/Drop Multiplexers. International Journal of Innovative Computing, Information and Control, 4(5): 1299-1313, 2008.

[3] Zahilah, R., et al. Lightpath Route Management System for IP-Over-CWDM Networks With ROADMS, Based On A ROADM Graph, International Journal of Innovative Computing, Information and Control, 7(5(A)): 2485-2501, 2011.

[4] Ziemann, O., P. Zamzow, and W. Daum. POF Handbook: Optical Short Range Transmission Systems, Springer, 2008. 
[5] Chandrappan, J., et al. Optical Coupling Methods for Cost-Effective Polymer Optical Fiber Communication, Components and Packaging Technologies, IEEE Transactions 32: 593-599, 2009.

[6] Haupt, M. and U.H.P Fischer. Design and development of a MUX/DEMUX element for WDM over POF, in International Students and Young Scientists Workshop in Photonics and Microsystems 2017: 27-31.

[7] Koonen, A.M.J., et al. Cost optimization of optical in-building networks, in 37th European Conference and Exhibition Optical Communication (ECOC) 2011: 1-3.

[8] Kagami, M. Visible Optical Fiber Communication, R\&D Review of Toyota CDRL. Toyota Central R\&D Labs, Japan, 2005.

[9] Imoto, K. et al. New biconically tapered fiber star coupler fabricated by indirect heating method. Journal of Lightwave Technology, 5(5): 694 - 699, 1987.

[10] David, S., F.M. Antonio, and J.A. Valenzuela. Fused fiber optics couplers, in The International Society for Optical Engineering Proceedings of SPIE p. 330-333 (2001).

[11] S. Ci-jun, D. Ji-an and Z. Jue. Development of a Novel Optical Fiber Coupler, in Sixth International Conference on Intelligent Systems Design and Applications 2006, pp: 183-186.

[12] Pal, B.P. Fabrication and Modeling of Fused Biconical Tapered Fiber Couplers. Fiber and integrated optics, 22(2): 97-117, 2003.

[13] Diemeer, M.B.J., W.J. DeVries and K.W. Benoist. Fused coupler switch using a thermo-optic cladding. Electronics Letters, 24(8): 457-458, 1988.

[14] Ab-Rahman, M.S., H. Guna and M.H. Harun. $1 \times$ N Self-Made Polymer Optical Fiber Based Splitter for POF-650 nm-LED based Application. in International Conference on Electrical Engineering and Informatics 2009, Selangor, Malaysia.

[15] Rosco, ROSCOLUX Color Filter, Rosco, Editor. Rosco: Harbor View Avenue, Stamford. pp. 1-2, 2003.

[16] Rosco, Roscolux, in 1991-2010. Rosco Laboratories, 2010.

[17] Appajaiah, A., V. Wachtendorf and W. Daum, Climatic exposure of polymer optical fibers: Thermooxidative stability characterization by chemiluminescence. Journal of Applied Polymer Science, 103(3): 1593-1601, 2007.

[18] Kuzyk, M. Polymer fiber optics: materials, physics, and applications, CRC/Taylor \& Francis, 2007.

[19] Bosc, D. Thermo-optical coefficient determination of index liquids used for optimization of optical integrated components. Optics Communications, 194(4-6): 353-357, 2001. 
[20] Kim, K.-T and K.-H. Park. Fiber-Optic Temperature Sensor Based on Single Mode Fused Fiber Coupler. J. Opt. Soc. Korea, 12: 152-156, 2008.

[21] Zhao, L.J., et al., Wide-Range Temperature Dependence of Brillouin Shift in Optical Fiber, in 1st IEEE International Conference-Nano/Micro Engineered and Molecular Systems. NEMS 2006. pp. 1327-1330.

[22] Yang, S.-W and H.-C. Chang. Numerical modeling of weakly fused fiber-optic polarization beam splitters. I. Accurate calculation of coupling coefficients and form birefringence. Journal of Lightwave Technology, 16(4): 685, 1998.

[23] Appajaiah, A. Climatic Stability of Polymer Optical Fibers. Potsdam University: Berlin. pp. 13-31, 2004.

[24] Hsieh, C.S., T.L. Wu and W.H. Cheng. Optimum approach for fabrication of low loss fused fiber couplers. Materials Chemistry and Physics, 69(1): 199-203, 2001.

[25] Zieamann, O., et al., POF Handbook: Optical Short Range Transmission Systems. 2nd ed. Berlin: Springer, 2008.

[26] Lutz, D., et al. Wavelength Division Multiplex Instructional Lab System with Polymeric Fibers for use in Higher Education. in Proceedings of the Symposium on Photonics Technologies for 7th Framework Program 2006, Wroclaw. 